\title{
Resultados preliminares do manejo integrado do fogo nas terras indígenas Paresi
}

\author{
Ananda Santa Rosa ${ }^{\mathrm{a}^{*}}$, Raquel Antunes Daldegan ${ }^{\mathrm{b}}$, Ruth Elias de Paula Laranja $^{\mathrm{a}}$, Rafael Rodrigues da Franca ${ }^{\mathrm{a}}$ \\ ${ }^{\text {a }}$ Universidade de Brasília (UnB), Brasil \\ ${ }^{\mathrm{b}}$ Universidade de Porto (UP), Portugal \\ * Autor correspondente (ananda.andrade@ aluno.unb.br)
}

\section{N F O}

\section{Keywords}

fire regime

vegetation damage

active fire

management

\begin{abstract}
A B S T R A C T
Preliminary results of the integrated fire management in Paresi indigenous lands

Integrated fire management considers local practices, ecological and scientific information, and institutional management to conserve biodiversity, reduce the prevalence of uncontrolled fire, and protect firesensitive vegetation. In indigenous lands, management is administered through Forest Brigades Program, which is supported by the Technical Cooperation Agreement between the Brazilian Institute of Environment and Renewable Natural Resources and the National Indian Foundation. There are still few evaluations on the implementation of this paradigm in Indigenous Lands focusing on the fire regime and the damage caused in the plant species, considering the periods of the fire treatment. Therefore, the objective of this work was to evaluate the first results of the integrated fire management in the Juininha, Paresi and Utiariti, Indigenous Lands, all occupied by the indigenous Paresi people and is located in the state of Mato Grosso, by analyzing, in 2016 to 2018 , the phytophysiognomies affected by burning, of vegetation damage caused by fire, and seasonal distribution of the active fire data from 2016 to 2018 . The results showed that burns on indigenous lands were shifted to the early fire season and that most of the evaluated species did not have damage caused by the burns of both periods. When with some impact level, this was higher in the period of early fire. Therefore, it was concluded that although burns are shifted to the early fire period, the prescribed burn techniques do not cover the frequency and history of the surfaces hit to consider a mosaic approach beyond the reduction of major events in the modal-late fire season.
\end{abstract}

\section{R E S U M O}

O manejo integrado do fogo considera as práticas locais, as informações ecológicas e científicas e o manejo institucional para conservar a biodiversidade, reduzir a prevalência de incêndios florestais e proteger a vegetação sensível ao fogo. Nas terras indígenas, a gestão do manejo ocorre por meio do Programa de Brigadas Florestais, que é respaldado pelo Acordo de Cooperação Técnica entre o Instituto Brasileiro do Meio Ambiente e dos Recursos Naturais Renováveis e pela Fundação Nacional do Índio. Ainda são escassas avaliações sobre a implementação deste paradigma em Terras Indígenas com enfoque ao regime de queima e aos danos causados em espécies vegetais, considerando os períodos de tratamento de fogo. Por isto, o objetivo deste trabalho foi avaliar os primeiros resultados do manejo integrado do fogo nas terras indígenas Juininha, Paresi e Utiariti, todos ocupadas pelo povo indígena Paresi e localizadas no estado do Mato Grosso, por meio da análise das fitofisionomias afetadas pelas queimas, dos danos na vegetação causadas pelo fogo e pela distribuição sazonal do dado de fogo ativo, nos anos de 2016 a 2018. Os resultados apresentaram que as queimas nas terras indígenas foram deslocadas para a estação do fogo precoce e que a maioria das espécies avaliadas não tiveram danos causados pelas queimas de ambos os períodos. Quando com algum nível de impacto, este foi mais elevado no período de fogo precoce. Por isto, concluiu-se que apesar das queimas estarem deslocadas para o período de fogo precoce, as técnicas de queimas prescritas não abrangem a frequência e histórico das superfícies atingidas para considerar uma abordagem de mosaicos além da redução dos grandes eventos na estação de fogo modal-tardio. 


\section{INTRODUÇÃO}

As queimadas naturais favoreceram a atual configuração do Cerrado (Ramos-Neto \& Pivello, 2000; Bownman et al., 2009) com fitofisionomias savânicas e campestres propensas a passagem do fogo quando, em muitos casos, relacionadas a um regime de queima natural que ocorre nos meses da estação chuvosa e de transição (Sato \& Miranda, 1996). Em razão do aumento da frequência e da recorrência das queimadas na estação seca (Batista et al., 2018), o padrão do fogo foi modificado (Pausas et al., 2004), bem como a resposta dos ecossistemas do Bioma. O motivo desta mudança é relacionado com a preponderância de um fogo antrópico ao natural. Como prova, estima-se que nos anos de 1900 a $2000,86 \%$ das queimadas originadas em Savanas são relacionadas com atividades antrópicas (Mouillot \& Field, 2005).

Para mitigar estes eventos, o Brasil adotou políticas proibitivas ao uso do fogo (Falleiro et al., 2016), sendo permitido apenas com uma licença ambiental específica ou para subsistência pelos povos indígenas ou se descrito no plano de manejo da unidade de conservação (Brasil, 2012). Apesar disto, as operações de combate aos incêndios continuam recorrentes, sendo gastos pelo poder público valores milionários para extingui-los em unidades de conservação e terras indígenas, conforme detalhamento das operações na plataforma Ciman Virtual (Centro Integrado Multiagências de Coordenação Operacional Nacional (Ciman), [2019]).

A implementação do Projeto de Prevenção, Controle e Monitoramento de Queimadas Irregulares e Incêndios Florestais no Cerrado (Projeto Cerrado Jalapão), em 2012, como parte da Iniciativa Internacional para a Proteção do Clima (IKI) e da cooperação bilateral entre Brasil e Alemanha, foi um marco para reduzir estes sinistros, aprimorar as metodologias de monitoramento e de atividades de campo e romper paradigmas sobre o uso do fogo por meio do programa piloto do manejo integrado do fogo (MIF) (Ministério do Meio Ambiente (MMA), [2017?]). Para cumprir estes escopos, um dos propósitos do Projeto foi incluir a realização de queimas prescritas no início da estação seca (Schmidt et al., 2016). Outros foram de considerar práticas locais, informações ecológicas, opções de gestão, criar mosaicos de paisagem de diferentes histórias de fogo, conservar a biodiversidade, reduzir a prevalência de incêndios florestais no período tardio, proteger a vegetação sensível ao fogo e reduzir os conflitos com os povos tradicionais (Myers, 2006; Schmidt et al., 2018).

No âmbito das Terras Indígenas (TIs), a gestão do MIF ocorre por meio do Programa de Brigadas
Federal (PBRIF), que é respaldado pelo Acordo de Cooperação Técnica (Fundação Nacional do Índio (Funai), 2013) entre o Instituto Brasileiro do Meio Ambiente e dos Recursos Naturais Renováveis (Ibama), por meio do Centro Nacional de Prevenção e Combate aos Incêndios Florestais (Prevfogo), e a Fundação Nacional do Índio (Funai). Por ele, a principal forma de envolvimento dos indígenas é com a contratação temporária destes como brigadistas, cuja celebração é aprovada pelo poder público. E.g.: autorizações referentes a 2019 (Ministério do Meio Ambiente (MMA), 2019); (Instituto Brasileiro do Meio Ambiente e dos Recursos Naturais Renováveis (Ibama), 2019). Outra forma é pela consulta, em especial com lideranças, anciões e mulheres, para entender o uso econômico e cultural do fogo e para identificar as práticas de queimadas tradicionais, de forma a subsidiar as estratégias do manejo (Centro Nacional de Prevenção e Combate aos Incêndios Florestais (Prevfogo), 2019). Estas atividades são baseadas nos saberes acumulados de geração em geração sobre a biodiversidade local e a resposta desta em relação ao emprego do fogo.

Estudos que corroboram e validam estas técnicas já são expressivos para unidades de conservação (ex. Schmidt et al. (2018), Borges et al. (2016) e Barradas (2017)). Para as terras indígenas, ainda são escassos, salvo o paper de Falleiro et al. (2016) que não considera o regime de queima e o impacto sobre a vegetação. Considerações da atuação da gestão pública e do MIF são importantes para analisar a continuidade das atividades que são realizadas para prevenir e combater incêndios em territórios tradicionais. Desta forma, o objetivo deste trabalho foi avaliar os primeiros resultados do manejo integrado do fogo nas terras indígenas Juininha, Paresi e Utiariti, todos ocupadas pelo povo indígena Paresi, nos anos de 2016 a 2018, por meio da análise das fitofisionomias afetadas, dos danos à vegetação causadas pelo fogo e pela distribuição sazonal do dado de fogo ativo.

\section{MATERIAL E MÉTODOS}

\section{As áreas de estudo}

As TIs Paresi, Juininha e Utiariti são habitadas por aproximadamente 1400 indígenas do povo $\mathrm{Pa}$ resi (Instituto Brasileiro de Geografia e Estatística (IBGE), 2010). Juntas, as três terras superam pouco mais de 1 milhão de hectares e estão situadas no oeste do estado do Mato Grosso. A maior proporção do território é composta por vegetação nativa, porém há usos para criação de gado, culturas de subsistência e produção econômica dos indígenas (Figura 1). 


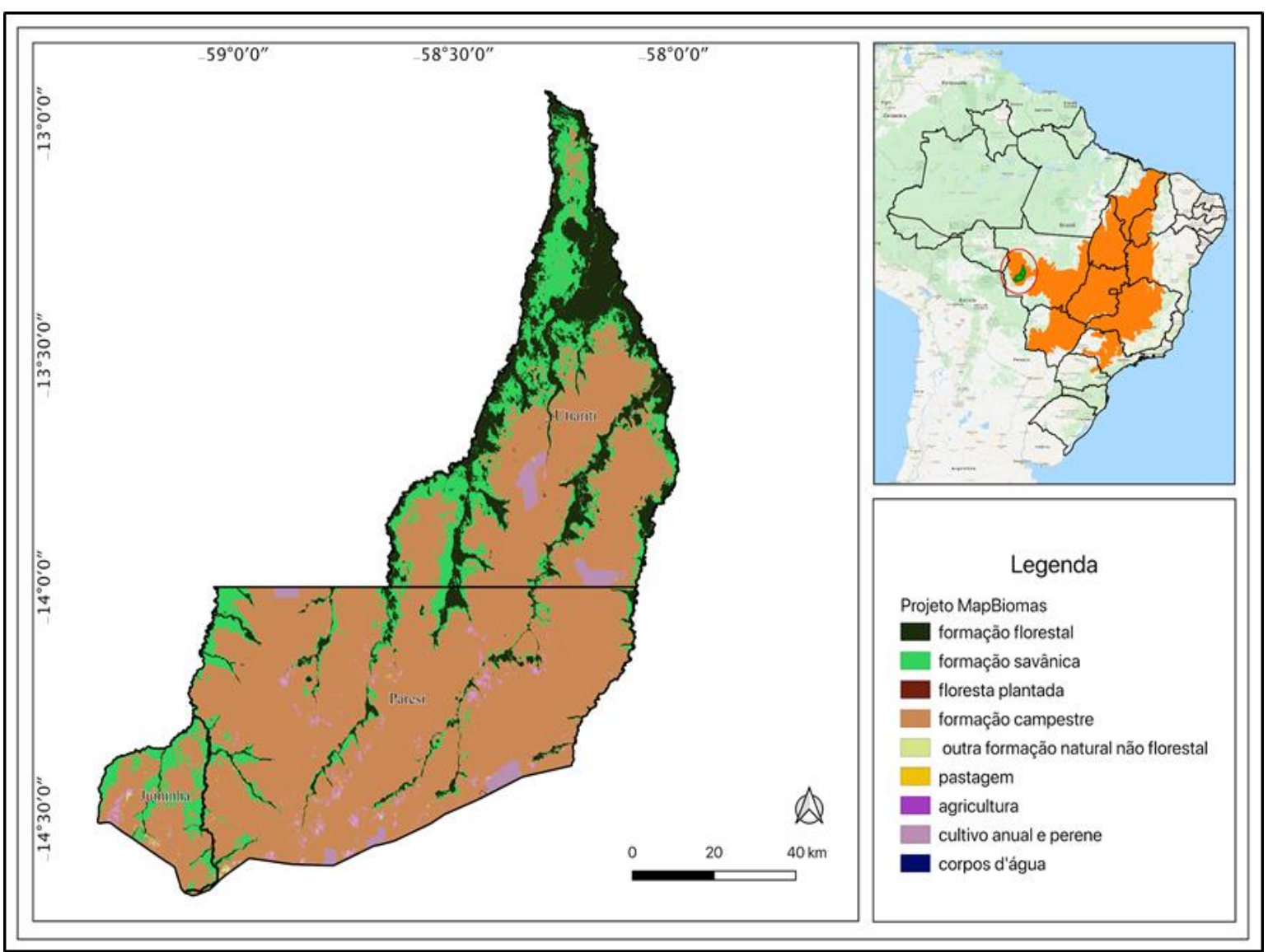

Figura 1 - Localização e principais tipos de vegetação e uso das terras indígenas Juininha, Paresi e Utiariti. As classificações da legenda, bem como a nomenclatura, são oriundas do mapa de 2018 do Projeto MapBiomas (2019).

O regime de chuvas na região é caracterizado por ser do tipo bimodal (Figura 2), sendo o primeiro período de ocorrência nos meses de janeiro a abril e o segundo de outubro a dezembro. Os meses mais secos, portanto, intercorre de maio a setembro.

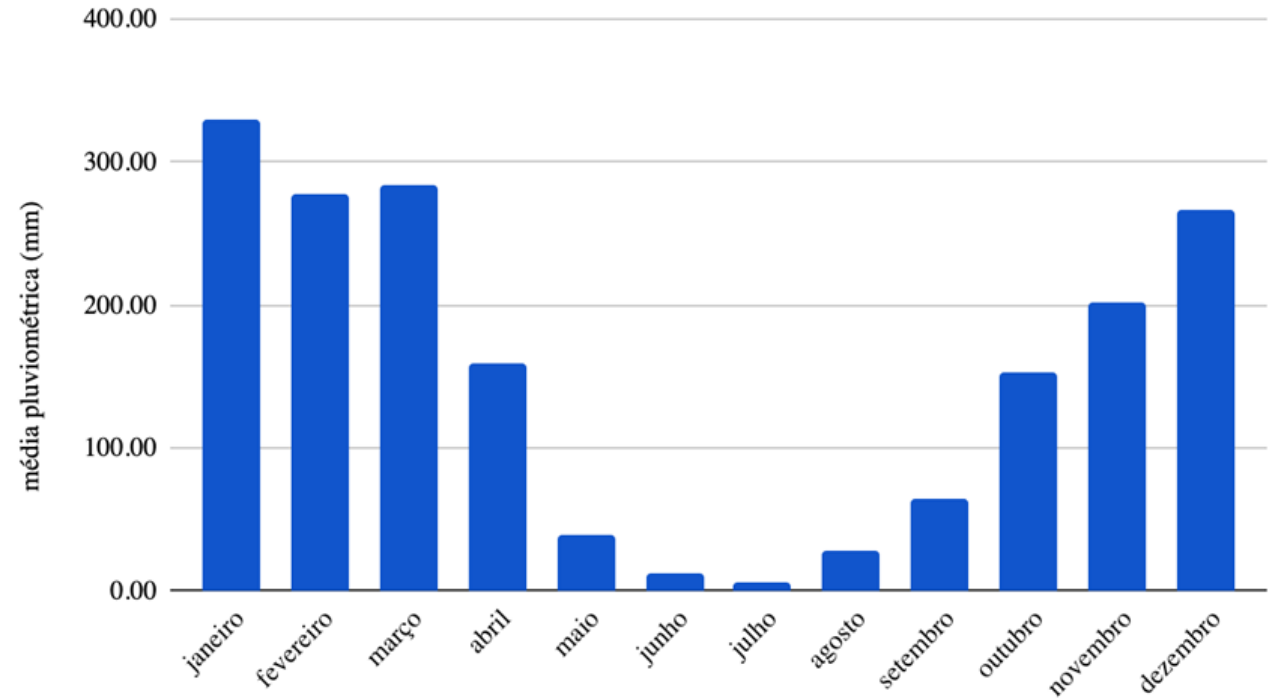

Figura 2 - Climatologia de precipitação da região. Esta climatologia de precipitação foi considerada a partir da média mensal dos dados registrados de 1983 a 2018 de uma estação pluviométrica localizada no centro da TI Utiariti (estação Bacaval - código 1358001). Os dados estão disponíveis para download na plataforma Hidroweb (da Agência Nacional de Águas (ANA), [2019]). 
Impactos socioambientais e culturais também são recorrentes nas áreas, como a contaminação por agrotóxicos (Soares et al., 2017) e pelas queimadas (Daldegan et al., 2019). Ainda que os indígenas façam uso tradicional do fogo (Prevfogo, 2019), a incidência das queimadas no período de estiagem é uma fonte de preocupação para os órgãos ambientais. Por isto, desde 2013 a região é contemplada pelo Programa de Brigadas Federal. A partir de 2016, houve a implementação do MIF com alocação de recursos e agentes especializados para realização de consultas aos indígenas e queimas prescritas de abril a junho.

\section{Análise dos Dados}

\section{Regime das queimas e vegetação afetada}

Os fogos ativos registrados nos anos de 2016 a 2018, pelo satélite VIIRS, coleção 01 (Schroeder et al., 2014), foram utilizadas para avaliar a variabilidade do fogo a partir do quantitativo total detectado por ano e nos períodos de tratamentos intra-anuais que são relacionados com as estações de chuva e seca: de fogo precoce (janeiro a junho e outubro a dezembro) e de fogo modal-tardio (compreendendo os meses de julho a setembro).

Para a identificação das fitofisionomias atingidas nestes períodos, foram utilizados os mapas, de resolução de $30 \mathrm{~m}$, dos anos de 2016, 2017 e 2018, do Projeto MapBiomas (Projeto Mapbiomas, 2019). Os mapas foram reamostrados para a mesma resolução espacial do dado de fogo ativo, $375 \mathrm{~m}$, através do método de pixels majoritários. Este procedimento permitiu, embora diminua a resolução espacial dos mapas de vegetação, o uso simultâneo de dados obtidos de diferentes sensores e resoluções espaciais.

\section{Análise do impacto da vegetação pós fogo}

Os dados de avaliação dos danos à vegetação pós queima foram obtidos com o Prevfogo/Ibama e divididos conforme períodos de tratamento. As avaliações foram realizadas em agosto e setembro de 2018 e inventariadas por brigadistas contratados, capacitados e habilitados metodologicamente para identificar e qualificar visualmente o impacto causado pelo fogo às espécies arbóreas adultas do Cerrado stricto sensu que são compreendidas pelo Prevfogo/Ibama de importância econômica e alimentar ao povo Paresi (Tabela 1).

Tabela 1 - Espécies vegetais avaliadas pós fogo em atividades de campo.

\begin{tabular}{lcccc}
\hline \multicolumn{1}{c}{ Família } & Nome científico & Nome popular & Nome Paresi & $\begin{array}{c}\text { Total de indivíduos } \\
\text { avaliados }\end{array}$ \\
\hline Apocynaceae & Hancornia speciosa & Mangaba & Kathyola & 1.722 \\
\hline Malpighiaceae & Brysonima sp. & Murici & Fare & 1.016 \\
\hline Caryocaraceae & Caryocar brasiliense & Pequi & Kani & 1.726 \\
\hline Sapotaceae & Pouteria ramiflora & Curiola ou Veadeira & Koma & 3.613 \\
\cline { 2 - 5 } & Pouteria torta & $\begin{array}{c}\text { Amarelinho, Guapeba } \\
\text { ou Abiú do cerrado }\end{array}$ & Manakata & 3.024 \\
\hline
\end{tabular}

Do banco disponibilizado, para os anos de 2016 a 2018, foram avaliados 11101 indivíduos, em 51 áreas (Figura 3), que não fazem parte de tratamentos de pesquisa e foram identificadas aleatoriamente com subsídio dos dados de fogo ativo dos satélites AQUA, TERRA e VIIRS do Banco Fire Information for Resource Management System (National Aeronautics and Space Administration (NASA), [2019]). 


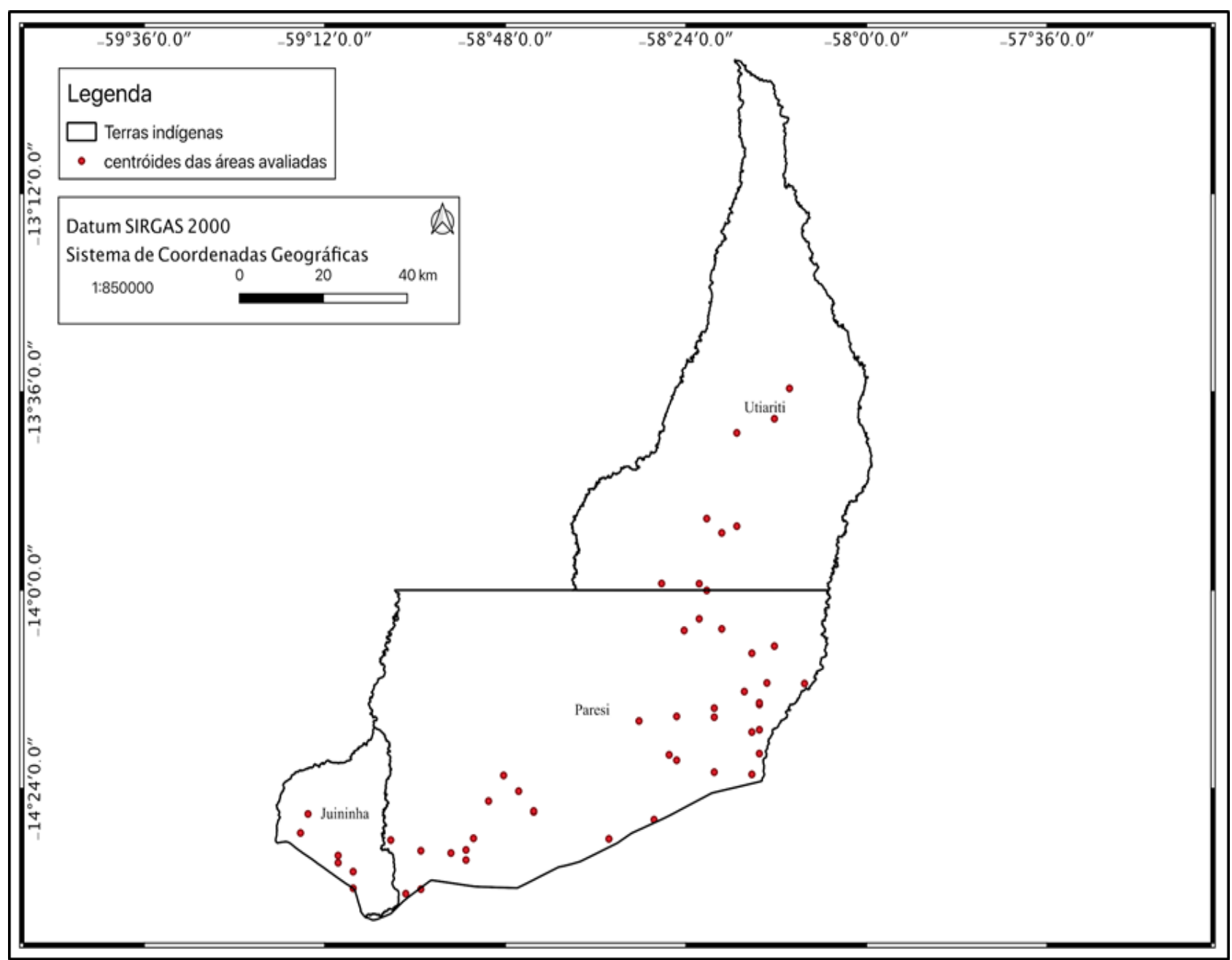

Figura 3 - Áreas onde ocorreram avaliação das queimadas. Estas não fazem parte de tratamento de pesquisa e foram identificadas aleatoriamente com o apoio de dados de fogo ativo.

Durante as avaliações, os danos foram classificados conforme percentual do perecimento das copas das árvores, dos caules, das flores e dos frutos relacionados a passagem do fogo (Tabela 2).

Tabela 2 - Classificação dos danos conforme perecimento da árvore.

\begin{tabular}{lc}
\hline Dano & Porcentagem \\
\hline Muito baixo & 0 a 20 \\
Baixo & 21 a 40 \\
Médio & 41 a 60 \\
Alto & 61 a 80 \\
Muito alto & 81 a 99 \\
Morte total da planta & 100 \\
\hline
\end{tabular}

\section{RESULTADOS E DISCUSSÃO}

\section{O padrão das queimadas pós implementação do MIF}

$\mathrm{O}$ fogo nas terras indígenas teve uma mudança na temporalidade das ocorrências: as detecções foram antecipadas para a temporada de fogo precoce (Figura 4). Partindo de 2016, quando grande parte dos fogos ativos detectados foram na estação de fogo modal-tardio, 53\% do total do ano, houve um incremento anual da proporção das detecções relacionadas às queimas prescritas e uma diminuição proporcional das ocorrências detectadas no período mais seco e crítico do ano (fogo modal-tardio). 


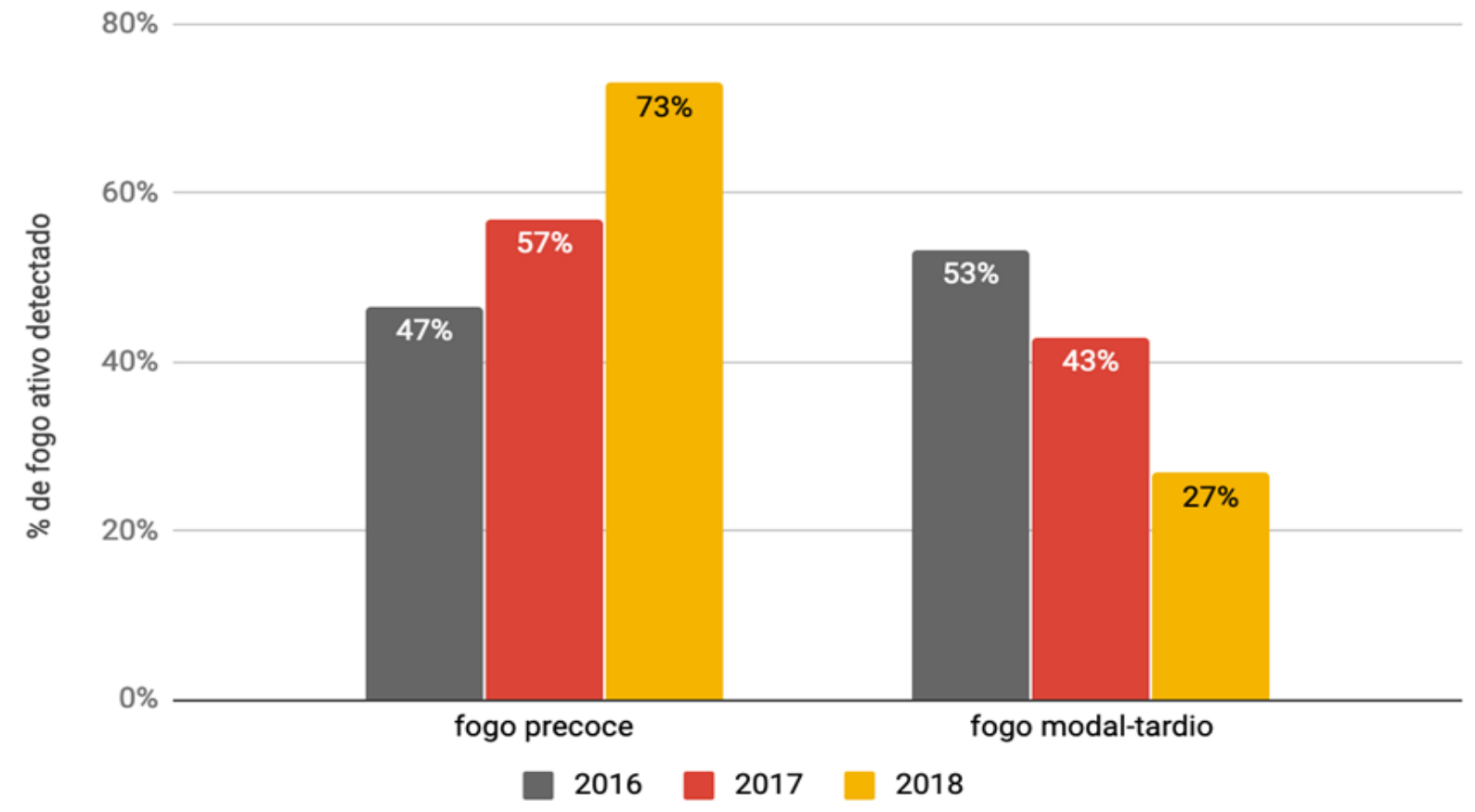

Figura 4 - Porcentagem anual de detecções de fogo ativo nos períodos MIF e estiagem.

Em relação às detecções mensais, estas foram concentradas de maio e junho, justamente nos meses de realização de queimas prescritas (Figura 5).
Durante o período modal-tardio, no entanto, ainda persistiram detecções significativas.

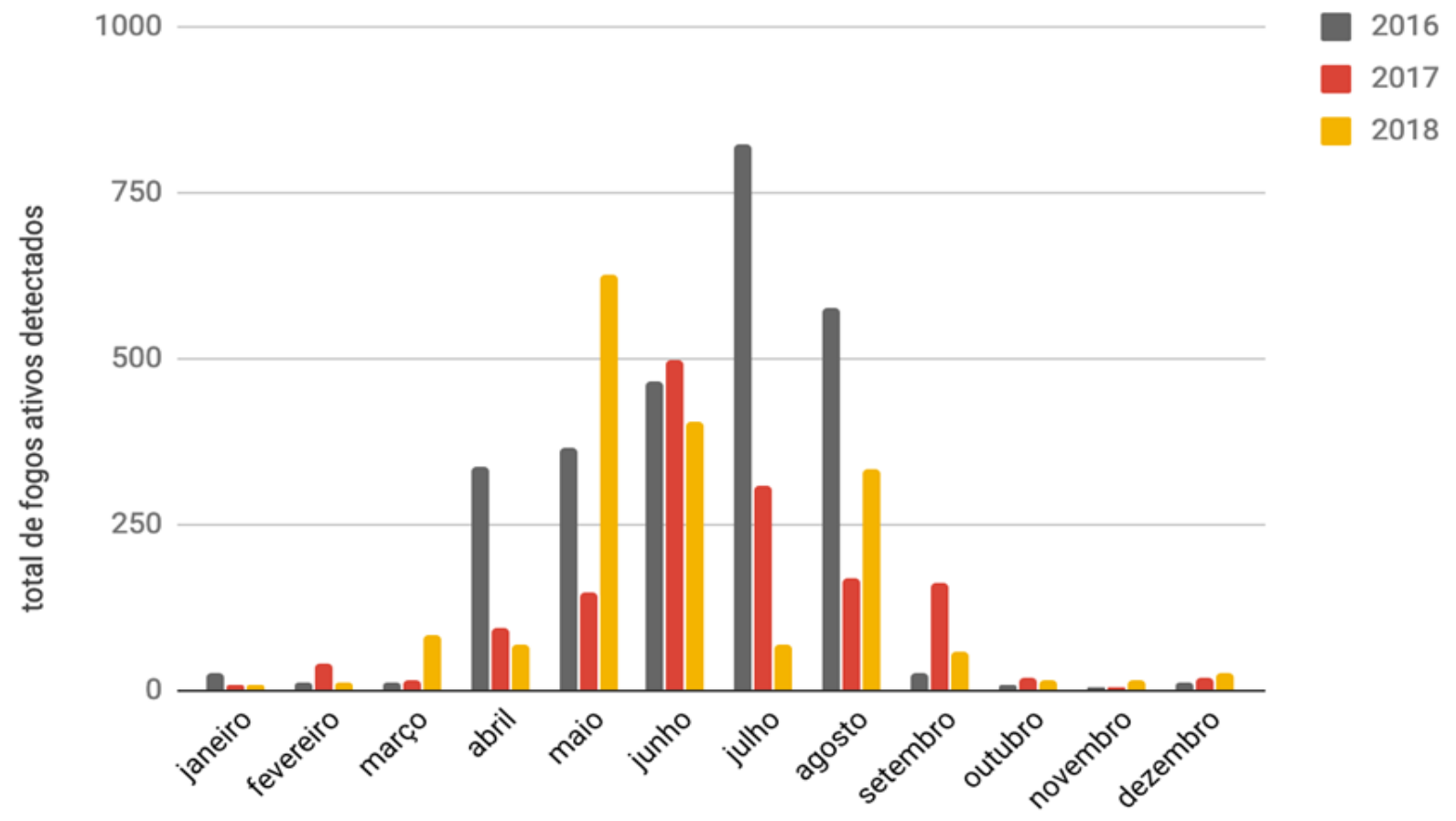

Figura 5 - Total de fogos ativos mensais, por ano. A quantidade total de focos detectados em 2016, 2017 e 2018 foram, respectivamente, 2672, 1485 e 1723. 
Quanto as fitofisionomias atingidas, o predomínio foi em vegetações propensas ao fogo-formações savânicas e campestres - para todos os anos e tratamentos. Nas formações florestais a proporção de áreas afetadas foi bastante reduzida (Tabela 3).

Tabela 3 - Porcentagem de detecção de fogos ativos por ecossistemas vegetais sensíveis (formações florestais) e propensos (formações savânicas e campestres) ao fogo.

\begin{tabular}{cccc}
\hline \multirow{2}{*}{ Ano } & Período & Formação florestal (\%) & $\begin{array}{c}\text { Formações savânicas e } \\
\text { campestres (\%) }\end{array}$ \\
\hline \multirow{2}{*}{2016} & Fogo precoce & 0,41 & 99,59 \\
& modal-tardio & 2,33 & 97,67 \\
\hline \multirow{2}{*}{2017} & Fogo precoce & 1,65 & 98,35 \\
& Fogo modal-tardio & 1,74 & 98,26 \\
\hline \multirow{2}{*}{2018} & Fogo precoce & 0,49 & 99,51 \\
& Fogo modal-tardio & 2,02 & 97,98 \\
\hline
\end{tabular}

Convém mencionar que não há base teórica e empírica para queima de florestas com a finalidade de proteção, uma vez que são sensíveis ao fogo (Myers, 2006). Além disso, apesar dos baixos valores registrados, estes podem estar relacionados a reamostragem dos mapas do Projeto MapBiomas (2019), um erro de decisão de manejo ou uma medida extremada para proteção de vastas áreas de incêndios. As queimas prescritas também podem ter sido baseadas nas práticas de manejo indígenas, guiadas pela cultura, economia ou entendimentos dos ciclos e do regime do fogo que não coadunam com o entendimento ecológico atual.

\section{Danos às espécies arbóreas}

A maioria das espécies avaliadas não foram impactadas diretamente pelo fogo para ambos os períodos; entretanto, quando com algum nível de degeneração, há sobreposição entre as distribuições de dano entre as queimas precoces e modal-tardia (perceptível pelas barras de erros das figuras 6, 7,8 e 9) e são discretamente mais elevados no período de fogo precoce.

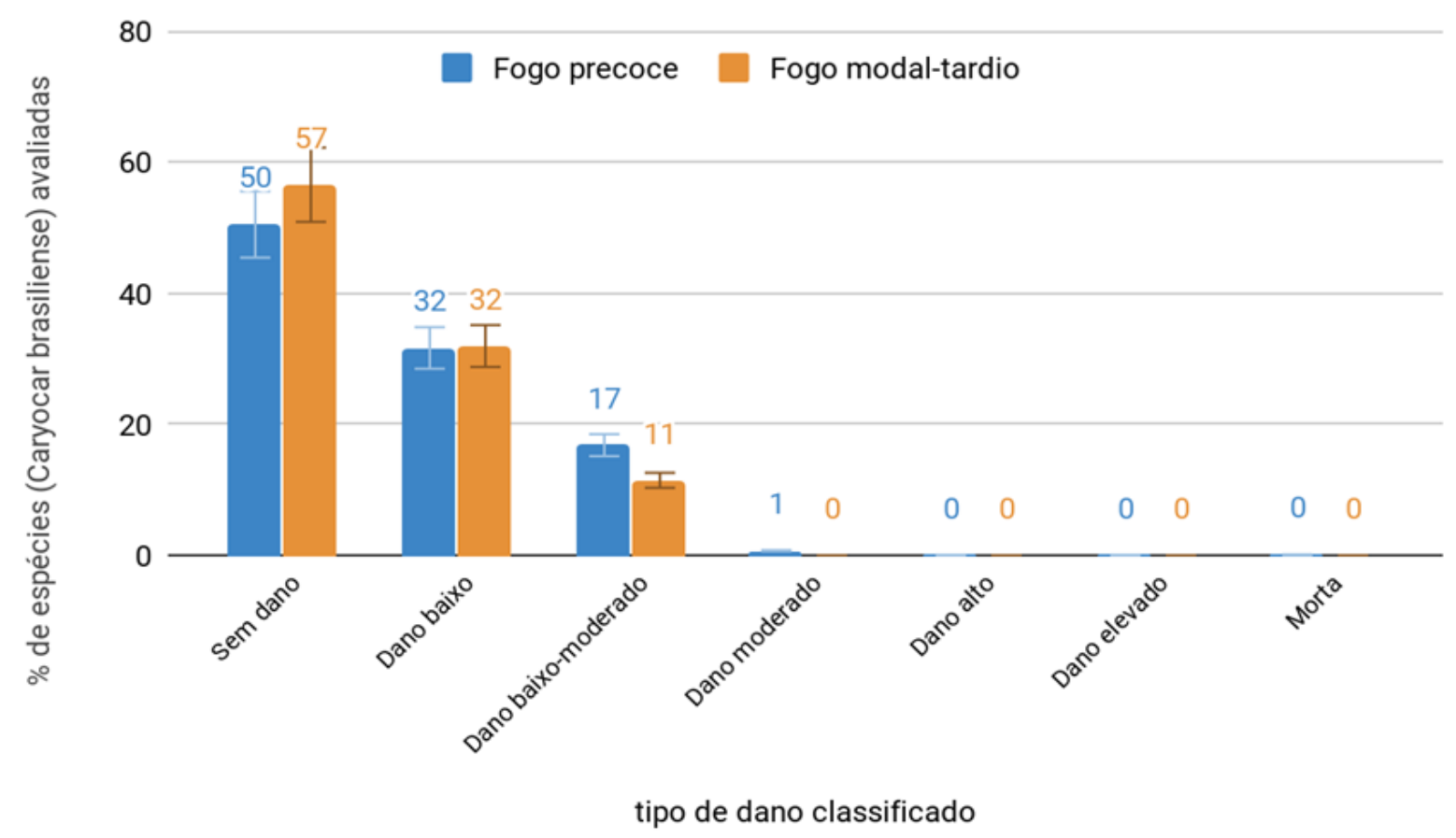

Figura 6 - Porcentagem dos danos de Caryocar brasiliense para as avaliações das áreas queimadas nos períodos de tratamento fogo precoce e fogo modal-tardio. 


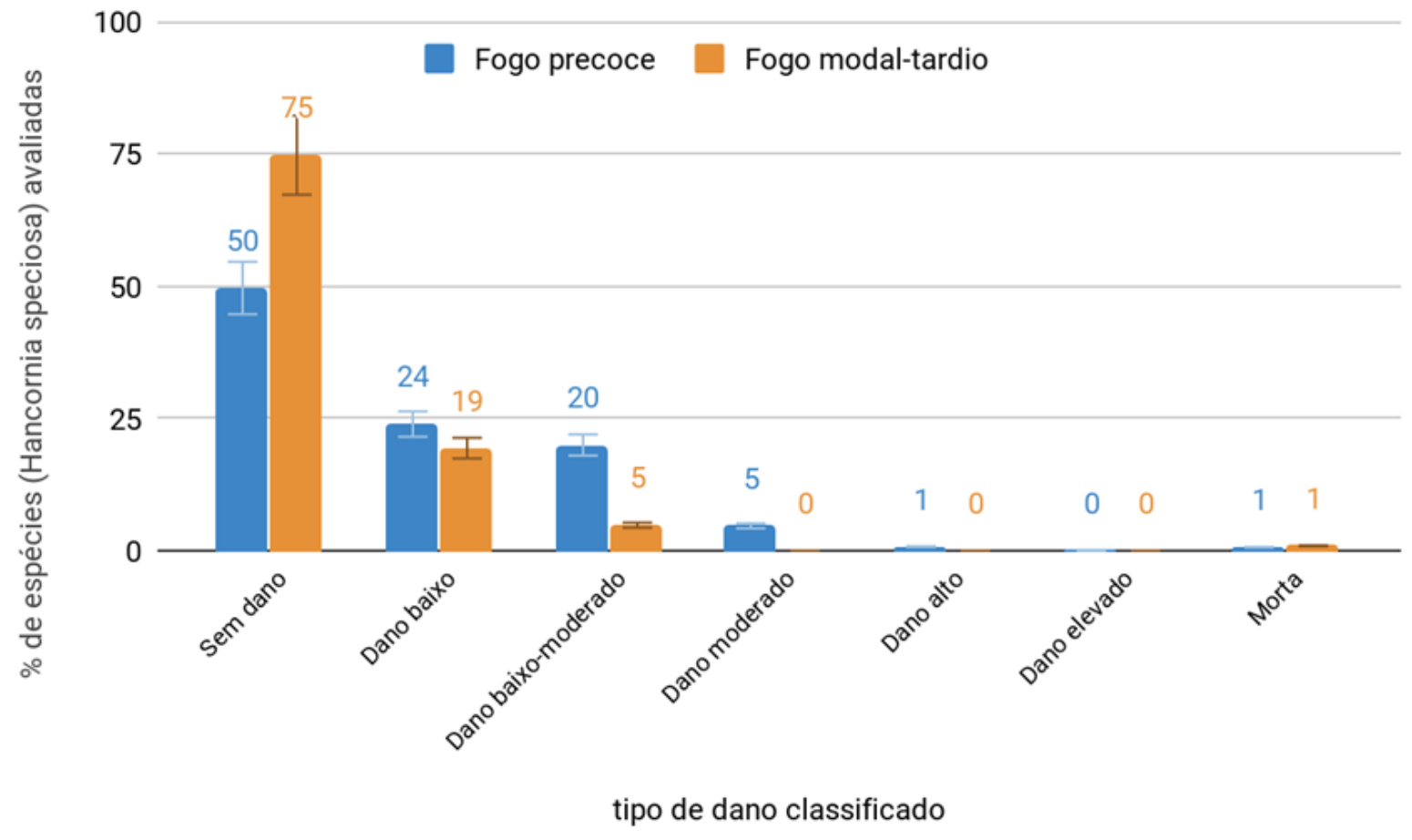

Figura 7 - Porcentagem dos danos de Hancornia speciosa para as avaliações das áreas queimadas nos períodos de tratamento do fogo precoce e fogo modal-tardio.

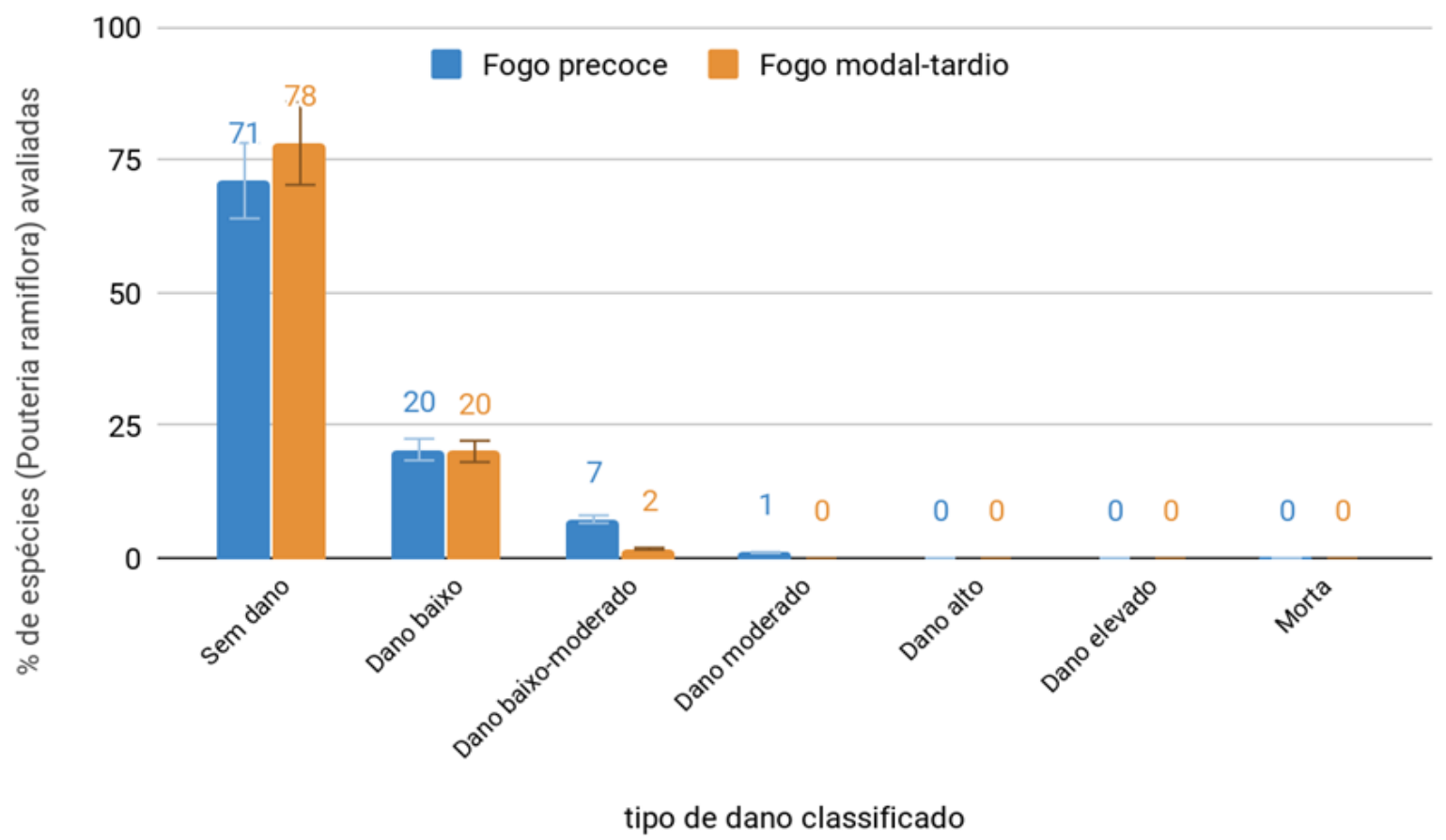

Figura 8 - Porcentagem dos danos de Pouteria ramiflora para as avaliações das áreas queimadas nos períodos de tratamento do fogo precoce e fogo modal-tardio. 


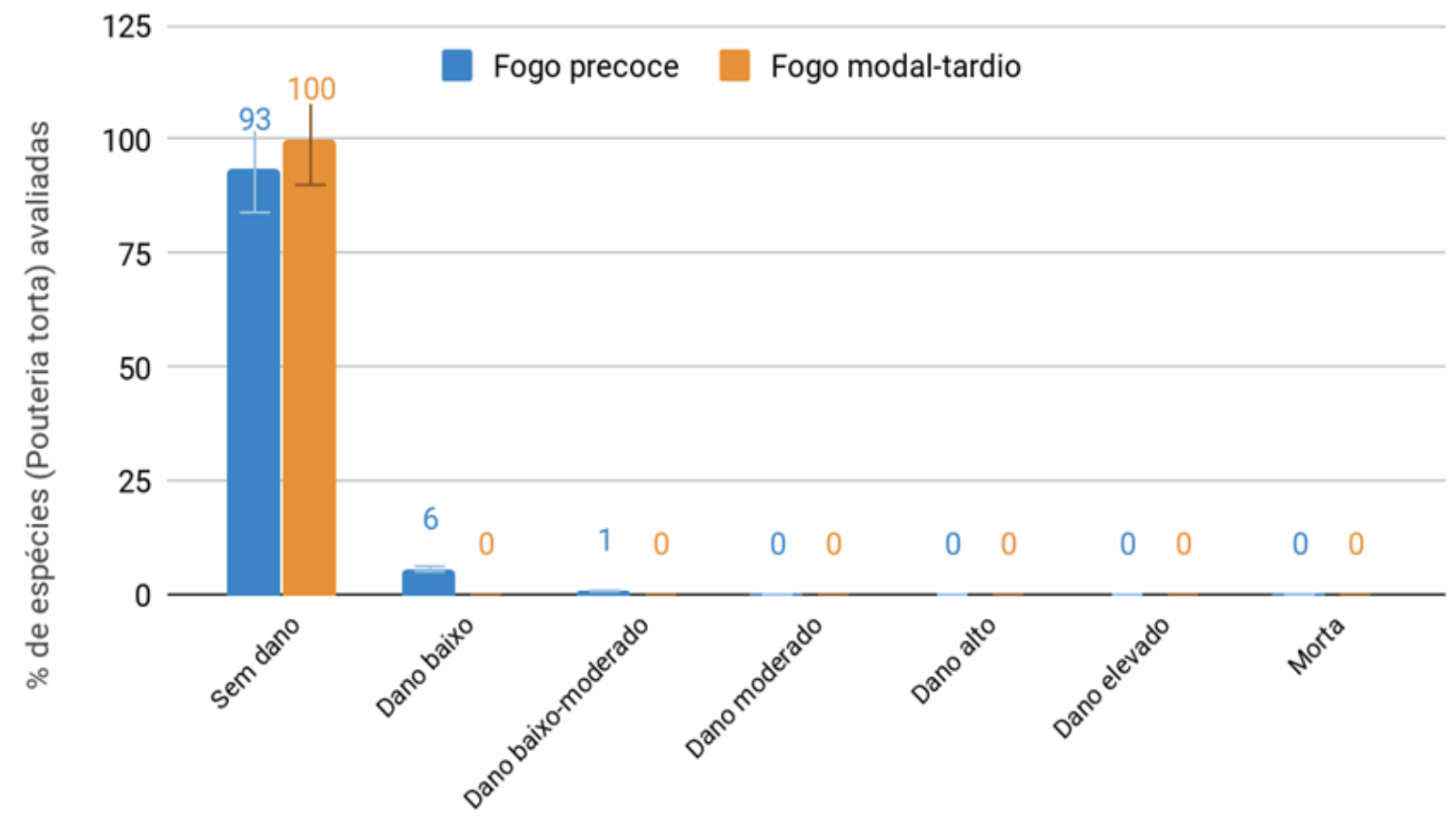

tipo de dano classificado

Figura 9 - Porcentagem dos danos de Pouteria torta para as avaliações das áreas queimadas nos períodos de tratamento do MIF e da estiagem.

Considerando trabalhos de Schmidt et al. (2016), Miranda et al. (2009) e Mistry et al. (2010), era esperado que os danos fossem menos severos no período de fogo precoce comparado com os resultados dos danos no período modal-tardio. Por isso, três pressupostos foram provocados para explicar este resultado: a primeira sobre a delimitação das áreas pelos agentes para a prescrição do fogo, relevando primariamente as áreas com a maior quantidade de matéria orgânica seca sem considerar a reincidência do fogo. A recorrência contínua das queimadas altera esta relação a partir de um segundo incidente, podendo refletir, geralmente em 10 anos, um grau de impacto de uma queimada do período de estiagem, mesmo que este fogo recorrente tenha sempre tratamento no período de fogo precoce (Sato, 2003).

A segunda é em relação a determinação da área sem prévia avaliação do tipo de queimada em ano pregresso. Possivelmente alguns danos podem ter sido agravados pela nova queima ou simplesmente oriundos de um evento antigo. A terceira é relativa a quantidade de espécies avaliadas e a metodologia empregada pelo Prevfogo para analisar danos em espécies arbóreas, vista a riqueza das comunidades em cada fitofisionomia e do emprego habitual de parcelas experimentais para tal finalidade (e.g.: Costa \& Rodrigues, 2015; Schmidt et al., 2016; Montenegro, 2019; Santana et al., 2019).

\section{O MIF é a resposta para reduzir os incêndios florestais e o impacto à biodiversidade nas Tis Paresi ?}

O manejo do fogo com os povos indígenas reorientou as políticas nacionais do fogo em consonância com o uso local e tradicional, a partir da dicotomia do fogo "bom", com base no conhecimento tradicional e aplicado com apoio de agentes especializados; e "ruim", sem elo com o conhecimento dos povos indígenas, descontrolado, de alta intensidade que provoca mortalidade de espécies da fauna e flora. Produzir o "fogo bom" requer condição e conhecimento antropológico sobre o povo em questão, em especial sobre a relação destes com os seus territórios. Isto vai muito além do entendimento exclusivo e restritivo sobre o uso da biodiversidade por estes povos (Ghosh \& Sahoo, 2011). Para além, este know-how também pode ser incompreendido ou erroneamente interpretado, já que usualmente existe uma ligação espiritual, religiosa e cultural considerados, em alguns casos, como elementos 
não científicos (Mercer et al., 2007). E.g.: Mistry et al. (2016) ao compreenderem o uso do fogo na terra indígena Xerente, estado do Tocantins, diagnosticaram que diferentes formas de plantio e uso do fogo estão atribuídas às fases da lua, descoincidente da metodologia de queimadas do agronegócio.

Outra reflexão indispensável é a validação das técnicas de avaliação de danos a vegetação que foram implementadas pelo Prevfogo/Ibama e a adoção de monitoramento (in locu e por satélite) das áreas em ambos os períodos, com enfoque à recorrência e à frequência do fogo, à quantidade de combustível acumulado e aos tipos de áreas com risco de queima em relação a evolução do deslocamento do fogo e de outros parâmetros, como a umidade relativa do ar, temperatura e velocidade do vento.

Ainda que estas queimas se sustentam pelas práticas e conhecimentos de manejo do Prevfogo, é igualmente importante avaliar os objetivos e intencionalidades destas, se associadas a práticas de manejos econômicos e institucionais ou aos valores culturais locais. Dentro do paradigma de manejo integrado do fogo, as queimas do segundo tipo são contempladas e vistas como legítimas. Caso não, especialmente quando atingem áreas florestadas (e está afastada de erros metodológicos de detecção e de análise geoespacial), suscita a atenção para estas práticas. Por isto, as políticas públicas precisam considerar outras esferas de observação e reconhecer que técnicas e empregos do fogo nem sempre são coincidentes/aplicáveis em uma escala nacional. Ressalta-se também a importância de incluir dados e informações de monitoramento para explicar o regime de queima, como produtos de áreas queimadas operacionais e com alta disponibilidade temporal para os usuários institucionais.

Neste sentido, para redução da quantidade de incêndios no período de fogo modal-tardio, o manejo integrado do fogo foi relevante nestas terras indígenas; entretanto, caso os danos resultantes das espécies avaliadas sejam em consequência da recorrência das queimas e sem base tradicional, o pressuposto do manejo do fogo para reduzir o combustível é uma prioridade com vias a homogeneização da paisagem e, por consequência, perda da biodiversidade.

\section{CONCLUSÕES}

As árvores que compõem as formações campestres e savânicas do Cerrado tem morfologia e fisiologia capazes de resistir relativamente bem a passagem do fogo e a estiagem sazonal; no entanto, há um limite para a resiliência destas espécies que, caso seja ultrapassado, há o agravamento de danos, independente da época de ocorrência, frequência e magnitude.
A adoção do MIF alterou em um curto espaço de tempo a época de ocorrência das queimas, antecipando-as para o período de fogo precoce. Ainda assim, foi notado que as técnicas de queimas prescritas não abrangem a frequência e histórico das superfícies atingidas para considerar uma abordagem de mosaicos além da redução dos grandes eventos no período de fogo modal-tardio.

Quanto ao impacto nas árvores avaliadas, os resultados aqui apresentados ainda são rudimentares e indicaram pouca diferença entre os danos causados à vegetação nos distintos períodos de tratamentos. Por essa razão, estes não são conclusivos, no que tange a redução de impactos ecológicos em consequência da pequena amostra, da possível metodologia de avaliação empregada pelo Prevfogo e da carência metodológica deste estudo que suscita análises estatísticas mais robustas.

\section{AGRADECIMENTOS}

O presente trabalho foi realizado com apoio do Conselho Nacional de Desenvolvimento Científico e Tecnológico (Processo n ${ }^{\circ}$ 13078/2019-1), da Coordenação de Aperfeiçoamento de Pessoal de Nível Superior - CAPES (Código de Financiamento 001) e do Centro Nacional de Prevenção e Combate aos Incêndios Florestais (Prevfogo/Ibama).

\section{REFERÊNCIAS BIBLIOGRÁFICAS}

Agência Nacional de Águas (ANA). Hidroweb - Downloads, [2019], acessado em 12/12/2019 através do link: http://www.snirh.gov.br/hidroweb/download

BARRADAS ACS. A gestão do fogo na Estação Ecológica Serra Geral do Tocantins, Brasil. Ano de obtenção: 2017. 135p. Dissertação (Mestrado Profissional em Biodiversidade em Unidades de Conservação) - Escola Nacional de Botânica/Jardim Botânico do Rio de Janeiro, Rio de Janeiro.

BATISTA EKL, RUSSELL-SMITH J, FRANÇA H, FIGUEIRA JEC. An evaluation of contemporary savanna fire regimes in the Canastra National Park, Brazil: Outcomes of fire suppression policies. Journal of Environmental Management, v. 205, p. 40-49, 2018.

https://doi.org/10.1016/j.jenvman.2017.09.053

BORGES SL, ELOY L, SCHMIDT IB, BARRADAS ACS, SANTOS, IA. Manejo do fogo em veredas: novas perspectivas a partir dos sistemas agrícolas tradicionais no jalapão. Ambiente \& Sociedade, v. 19, n. 3, p. 275-300, 2016. http://dx.doi.org/10.1590/18094422ASOC20150020R1V1932016

BOWMAN DM, BALCH JK, ARTAXO P, BOND WJ, CARLSON JM, COCHRANE MA, JOHNSTON FH. Fire in the Earth system. Science, v. 324, n. 5926, p 481-484, 2009.

https://doi.org/10.1126/science.1163886 
Brasil. Lei $\mathrm{n}^{\circ} 12.651$, de 25 de maio de 2012. Institui o Novo Código Florestal, 2012, acessado em 17/10/2019 através do link:

http://www.planalto.gov.br/ccivil_03/_ato20112014/2012/lei/l12651.html

Centro Integrado Multiagências de Coordenação Operacional Nacional (Ciman). Visão geral - Operações, [2019], acessado em 19/10//2019 através do link: http://queimadas.dgi.inpe.br/queimadas/ciman/operacoes/

Centro Nacional de Prevenção e Combate aos Incêndios Florestais (Prevfogo). Resumo expandido do relatório da compilação dos resgates do conhecimento tradicional sobre o uso do fogo em terras indígenas brasileiras. Brasília: Ibama, 34p. 2019.

COSTA YT, RODRIGUES, SC. Efeito do fogo sobre vegetação e solo a partir de estudo experimental em ambiente de cerrado. Revista do Departamento de Geografia, v. 30, p. 149-165, 2015.

DALDEGAN GA, ROBERTS D A, RIBEIRO FF. Spectral mixture analysis in Google Earth Engine to model and delineate fire scars over a large extent and a long time-series in a rainforest-savanna transition zone. Remote Sensing of Environment, v. 232, 2019.

https://doi.org/10.1016/j.rse.2019.111340.

FALLEIRO RM, SANTANA MT, BERNI CR. As contribuições do Manejo Integrado do Fogo para o controle dos incêndios florestais nas Terras Indígenas do Brasil. Biodiversidade Brasileira, v. 6, n. 2, p. 88-105, 2016.

Fundação Nacional do Índio (Funai). Firmado acordo entre Funai e Ibama para combater e prevenir incêndios em terras indígenas, 2013, acessado em 05/11/2019 através do link:

http://www.funai.gov.br/index.php/comunicacao/noticias/1060-firmado-acordo-entre-funai-e-ibama-para-combater-e-prevenir-incendios-em-terras-indigenas

GHOSH PK, SAHOO B. Indigenous traditional knowledge. Orissa Review, p. 65-70, 2011.

Instituto Brasileiro de Geografia e Estatística (IBGE). Censo 2010: Características Gerais dos Indígenas - Resultados do Universo, 2010, acessado em 13/09/2019 através do link: http://www.ibge.gov.br/home/estatistica/populacao/censo2010/caracteristicas_gerais_indigenas/default_caracteristicas_gerais_indigenas.shtm

Instituto Brasileiro do Meio Ambiente e dos Recursos Naturais Renováveis (Ibama). Portaria $\mathrm{n}^{\circ} 2.094$, de 6 de junho de 2019. Diário Oficial da União, Brasília, DF, ed. 110, seção 1, p. 62. 2019.

MERCER J., DOMINEY-HOWES, D, KELMAN, I, LLOYD K. The potential for combining indigenous and western knowledge in reducing vulnerability to environmental hazards in small island developing states. Environmental Hazards, v. 7, n. 4, p 245-256, 2007. https://doi.org/10.1016/j.envhaz.2006.11.001

Ministério do Meio ambiente (MMA). Projeto Cerrado Jalapão - Apresentação, [2017?], acessado em 13/11/2019 através do link:

http://cerradojalapao.mma.gov.br/projeto
Ministério do Meio Ambiente - MMA. Portaria No 153, de 18 de março de 2019. Diário Oficial da União, Brasília, DF, ed. 68, seção 1, p. 1242019.

MIRANDA HS, SATO MN, NETO WN, AIRES, FS. Fires in the cerrado, the Brazilian savanna. Tropical fire ecology. Berlin: Springer, p. 427-450, 2009.

MISTRY, J.; BILBAO, B. A.; BERARDI, A. Community owned solutions for fire management in tropical ecosystems: case studies from Indigenous communities of South America. Philosophical Transactions of the Royal Society B: Biological Sciences, v. 371, n. 1696, p. 1-10, 2016. https://doi.org/10.1098/rstb.2015.0174

MISTRY J, BERARD A, DURIGAN G. A influência do regime de fogo na variação estrutural em microescala e o mosaico da vegetação de Cerrado. Revista do Instituto Florestal, v. 22, n. 1, p. 33-49, 2010.

MONTENEGRO SR. Efeitos do manejo do fogo na estrutura de comunidades lenhosas em formações savânicas do Cerrado. Ano de obtenção: 2019. Dissertação (Mestrado em Ecologia) - Universidade de Brasília, Departamento de Pós Graduação em Ecologia, Brasília.

MOUILLOT F, FIELD CB. Fire history and the global carbon budget: a $1 \times 1$ fire history reconstruction for the 20th century. Global Change Biology, v. 11, n. 3, p 398-420, 2005.

https://doi.org/10.1111/j.1365-2486.2005.00920.x

MYERS RL. Convivendo com o Fogo - Manutenção dos ecossistemas e subsistência com o manejo integrado do fogo. Tallahassee USA: The Nature Conservancy - Iniciativa Global para o Manejo do Fogo, 36p. 2006.

National Aeronautics and Space Administration (NASA). Archive Download, [2019], acessado em 15/08/2018 através do link: https://firms.modaps.eosdis.nasa.gov/download/

PAUSAS JG, BRADSTOCK RA, KEITH DA, KEELEY JE. Plant functional traits in relation to fire in crown-fire ecosystems. Ecology, v. 85, n. 4, p. 1085-1100, 2004. https://doi.org/10.1890/02-4094

Projeto MapBiomas. Projeto MapBiomas - Coleção 4 da Série Anual de Mapas de Cobertura e Uso de Solo do Brasil, 2019, acessado em 12/08/2019 através do link: https://mapbiomas.org/

RAMOS-NETO MB, PIVELLO VR. Lightning fires in a Brazilian savanna National Park: rethinking management strategies. Environmental management, v. 26, n. 6, p 675684, 2000. https://doi.org/10.1007/s002670010124

SANTANA TF, FERNANDES HE, GIONGO M, MOURA WS, CABRAL KP, SOUZA, PB. Influência Do Fogo Na Germinação De Três Espécies Do Bioma Cerrado. Biodiversidade, v. 18, n. 1, p 18-27, 2019.

SATO MN, MIRANDA HS. Mortalidade de plantas lenhosas do cerrado sensu stricto submetidas a diferentes regimes de queima. In: Impactos de Queimadas em Áreas de Cerrado e Restinga. Brasília: Universidade de Brasília, 1996.

SATO MN. Efeito a longo prazo de queimadas na estrutura da comunidade de lenhosas da vegetação do cerrado sensu 
stricto. Ano de obtenção: 2003. Tese (Doutorado acadêmico em Ecologia) - Universidade de Brasília, Departamento de Ecologia, Brasília.

SCHMIDT IB, FONSECA CB, FERREIRA MC, SATO MN. Implementação do programa piloto de manejo integrado do fogo em três unidades de conservação do Cerrado. Biodiversidade Brasileira, v. 6, n. 2, p. 55-70, 2016.

SCHMIDT IB, MOURA, LC, FERREIRA MC, ELOY L, SAMPAIO, AB., DIAS PA, BERLINCK, CN. FIRE management in the Brazilian savanna: First steps and the way forward. Journal of applied ecology, v. 55, n. 5, p 20942101, 2018.

https://doi.org/10.1111/1365-2664.13118

SCHROEDER W, OLIVA P, GIGLIO L, CSISZAR, IA. The New VIIRS $375 \mathrm{~m}$ active fire detection data product: Algorithm description and initial assessment. Remote Sensing of Environment, v. 143, p. 85-96, 2014.

https://doi.org/10.1016/j.rse.2013.12.008

SOARES DF, FARIA AM, ROSA AH. Análise de risco de contaminação de águas subterrâneas por resíduos de agrotóxicos no município de Campo Novo do Parecis (MT), Brasil. Engenharia Sanitária Ambiental, v. 22, n. 2, p 277 284, 2017.

https://dx.doi.org/10.1590/s1413-41522016139118 